\title{
Publisher Correction: Early preclinical detection of prions in the skin of prion-infected animals
}

\author{
Zerui Wang 1,2, Matteo Manca ${ }^{3}$, Aaron Foutz ${ }^{4}$, Manuel V. Camacho1, Gregory J. Raymond ${ }^{3}$, Brent Race ${ }^{3}$, \\ Christina D. Orru ${ }^{3}$, Jue Yuan ${ }^{1}$, Pingping Shen ${ }^{1,2}$, Baiya Li, ${ }^{1,5}$, Yue Lang ${ }^{1,2}$, Johnny Dang ${ }^{1}$, Alise Adornato ${ }^{1}$, \\ Katie Williams ${ }^{3}$, Nicholas R. Maurer ${ }^{1}$, Pierluigi Gambetti ${ }^{1}$, Bin $\mathrm{Xu}^{6}$, Witold Surewicz ${ }^{7}$, Robert B. Petersen ${ }^{1,8}$, \\ Xiaoping Dong9 ${ }^{9}$, Brian S. Appleby ${ }^{1,4,10}$, Byron Caughey (1) ${ }^{3}$, Li Cui ${ }^{2}$, Qingzhong Kong ${ }^{1,4,10,11}$ \& \\ Wen-Quan Zou'12,4,9,10,11
}

Correction to: Nature Communications; https://doi.org/10.1038/s41467-018-08130-9; Published online Jan 162019.

The original version of this Article contained errors in the author affiliations.

Affiliation 2 incorrectly read 'Department of Neurology, The First Hospital of Jilin University, Changchun 130021 Jilin Province, China.'

Affiliation 5 incorrectly read 'Department of Otolaryngology, The First Affiliated Hospital of Xi'an Jiaotong University, Xi'an 710061 Shanxi Province, China'

Affiliation 9 incorrectly read 'State Key Laboratory for Infectious Disease Prevention and Control, National Institute for Viral Disease Control and Prevention, Chinese Center for Disease Control and Prevention, Beijing 102206, China.'

This has now been corrected in both the PDF and HTML versions of the Article.

Published online: 04 February 2019

\footnotetext{
${ }^{1}$ Department of Pathology, Case Western Reserve University School of Medicine, Cleveland 44106 OH, USA. ${ }^{2}$ Department of Neurology, The First Hospital of Jilin University, Changchun 130021 Jilin Province, The People's Republic of China. ${ }^{3}$ Laboratory of Persistent Viral Diseases, Rocky Mountain Laboratories, National Institute of Allergy and Infectious Diseases (NIAID), National Institutes of Health (NIH), Hamilton 59840 MT, USA. ${ }^{4}$ National Prion Disease Pathology Surveillance Center, Case Western Reserve University School of Medicine, Cleveland 44106 OH, USA. ${ }^{5}$ Department of Otolaryngology, The First Affiliated Hospital of Xi'an Jiaotong University, Xi'an 710061 Shanxi Province, The People's Republic of China. ${ }^{6}$ Department of Biochemistry, Virginia Polytechnic Institute and State University, Blacksburg 24061 Virginia, USA. ${ }^{7}$ Department of Physiology and Biophysics, Case Western Reserve University School of Medicine, Cleveland 44106 OH, USA. ${ }^{8}$ Foundational Sciences, Central Michigan University College of Medicine, Mount Pleasant 48859 MI, USA. ${ }^{9}$ State Key Laboratory for Infectious Disease Prevention and Control, National Institute for Viral Disease Control and Prevention, Chinese Center for Disease Control and Prevention, Beijing 102206, The People's Republic of China. ${ }^{10}$ Department of Neurology, University Hospitals Cleveland Medical Center, Case Western Reserve University School of Medicine, Cleveland 44106 OH, USA. ${ }^{11}$ National Center for Regenerative Medicine, Case Western Reserve University School of Medicine, Cleveland 44106 OH, USA. These authors contributed equally: Zerui Wang, Matteo Manca, Aaron Foutz, Manuel V. Camacho. Correspondence and requests for materials should be addressed to B.C. (email: bcaughey@nih.gov) or to L.C. (email: chuili1967@126.com) or to Q.K. (email: qxk2@case.edu) or to W.-Q.Z. (email: wxz6@case.edu)
} 